\title{
The random case of Conley's theorem*
}

\author{
Zhenxin Liu ${ }^{\dagger}$ \\ College of Mathematics, Jilin University, Changchun 130012, People's Republic of China
}

\begin{abstract}
The well-known Conley's theorem states that the complement of chain recurrent set equals the union of all connecting orbits of the flow $\varphi$ on the compact metric space $X$, i.e. $X-\mathcal{C R}(\varphi)=\bigcup[B(A)-A]$, where $\mathcal{C} \mathcal{R}(\varphi)$ denotes the chain recurrent set of $\varphi, A$ stands for an attractor and $B(A)$ is the basin determined by $A$. In this paper we show that by appropriately selecting the definition of random attractor, in fact we define a random local attractor to be the $\omega$-limit set of some random pre-attractor surrounding it, and by considering appropriate measurability, in fact we also consider the universal $\sigma$-algebra $\mathcal{F}^{u}$ measurability besides $\mathcal{F}$-measurability, we are able to obtain the random case of Conley's theorem.

Keywords: Random chain recurrence; Random local attractor; Random dynamical systems
\end{abstract}

\section{Introduction and main result}

Among the tasks of differential equations and dynamical systems, a fundamental one is to study qualitative, asymptotic, long-term behavior of solutions/orbits. Conley in his famous work [5] introduced the concept of chain recurrence, and defined an attractor to be the $\omega$-limit set of one of its neighbourhoods. He obtained the very interesting intrinsic relation between attractors and chain recurrent set. First we take a simple retrospect about his result.

Suppose $(X, d)$ is a compact metric space and $\varphi$ is a flow with the phase space $X$. An open nonempty set $U$ is called a pre-attractor for flow $\varphi$ if

$$
\overline{\bigcup_{t \geq T} \varphi(t, U)} \subset U
$$

for some $T>0$. In fact, $\overline{\varphi\left(T_{0}, U\right)} \subset U$ for some $T_{0}$ implies (1.1) holds, for details see page 33 of [5]. For the convenience of late use, we adopt the form (1.1.1). The attractor determined by the pre-attractor $U$ is defined by

$$
A:=\bigcap_{t \geq T} \overline{\bigcup_{s \geq t} \varphi(s, U)}
$$

It is easy to see that $A$ is a compact set, which is invariant under the flow $\varphi$, i.e. $\varphi(t, A)=$ $A, \forall t \in \mathbb{R}$. The basin of $A$, denoted by $B(A)$, is defined by

$$
B(A)=\{x \mid \varphi(t, x) \in U \text { for some } t \geq 0\} .
$$

Since $X$ is compact, it is obvious that $B(A)$ is independent of the choice of $U$. Therefore we denote it by $B(A)$, not mentioning $U$.

\footnotetext{
*Published in: Nonlinearity 19 (2006), 277-291.

${ }^{\dagger}$ E-mail address: zxliu@email.jlu.edu.cn (Zhenxin Liu).
} 

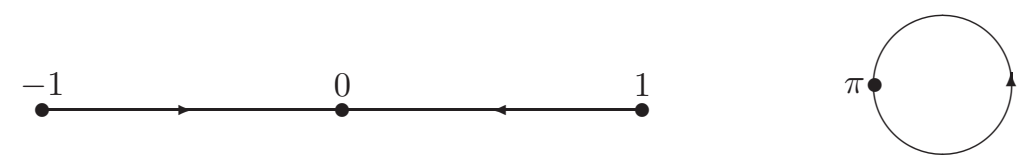

Figure 1: Chain recurrent sets for $\dot{x}=x^{3}-x$ and $\dot{\theta}=\cos ^{2} \frac{\theta}{2}$

For given $\epsilon, T>0$, a finite sequence $\left(x_{0}, t_{0}\right),\left(x_{1}, t_{1}\right), \cdots,\left(x_{n}, t_{n}\right)$ in $X \times(0, \infty)$ is called an $\epsilon$ - $T$-chain for $\varphi$ if

$$
d\left(\varphi\left(t_{j}, x_{j}\right), x_{j+1}\right)<\epsilon, t_{j} \geq T
$$

for $j=0,1, \cdots, n-1$. And we call $n$ the length of the chain. A point $p \in X$ is called chain recurrent if for any $\epsilon, T>0$, there is an $\epsilon$-T-chain with the length at least 1 which begins and ends at $p$. And we use $\mathcal{C R}(\varphi)$ to denote the set of all chain recurrent points in $X$.

Conley's theorem tells us that the complement of the chain recurrent set is in fact the union of the sets $B(A)-A$, as $A$ varies over the collection of attractors of $\varphi$, i.e.

$$
X-\mathcal{C R}(\varphi)=\bigcup[B(A)-A] .
$$

For example, consider the differential equation $\dot{x}=x^{3}-x$ on the interval $X=[-1,1]$ and assume $\varphi$ is the flow generated by it. Besides the two trivial attractors $\emptyset, X$, it is obvious that all other attractors are $\{0\},[-1,0]$ and $[0,1]$ with basins of attractions $(-1,1),[-1,1)$ and $(-1,1]$ respectively. Hence by $[1.3)$, we easily obtain that $\mathcal{C} \mathcal{R}(\varphi)=\{-1,0,1\}$, just the equilibria of the flow $\varphi$. See the left picture in Figure 1

Another simple example, consider $\dot{\theta}=\cos ^{2} \frac{\theta}{2}$ on $S^{1}$, and assume $\varphi$ is the flow generated by it. Then it is easy to see that there is no nontrivial attractors for $\varphi$, noticing that the unique equilibrium $\pi$ is not an attractor. So, by (1.3), the chain recurrent set for $\varphi$ is the whole circle $S^{1}$. See the right picture in Figure 1

Conley's result was adapted for maps on compact spaces by Franks [12, was later established for maps on locally compact metric spaces by Hurley [13] 14, and was extended by Hurley [15] for semiflows and maps on arbitrary metric spaces. In this paper, we will extend Conley's theorem to the random case, i.e. we will show that the similar result holds for cocycle $\varphi$ on compact metric spaces. For random dynamical systems, by defining random chain recurrent variable, which is the counterpart of chain recurrent point in random case, and by defining random attractor similar to (1.2), we obtain the main result of this paper, which states as follows:

Theorem 1 (Random Conley's theorem). Suppose $U(\omega)$ is an arbitrary random preattractor, $A(\omega)$ is the random local attractor determined by $U(\omega)$, and $B(A)(\omega)$ is the random basin determined by $A(\omega)$, then we have the following holds:

$$
X-\mathcal{C R}_{\varphi}(\omega)=\bigcup[B(A)(\omega)-A(\omega)],
$$

where the union is taken over all random local attractors determined by random pre-attractors, and "=" in 1.4) holds $\mathbb{P}$-almost surely.

Detailed definitions and notations in the main theorem can be found in the next section.

Similar to deterministic Conley's theorem, our result accurately describes where on earth the random chain recurrent variables lie. 


\section{Preliminaries}

In this section, we will give some preliminary definitions and propositions for the late use. Firstly we give the definition of continuous random dynamical systems (cf. Arnold [1).

Definition 2.1 A (continuous) random dynamical system (RDS), shortly denoted by $\varphi$, consists of two ingredients:

(i) A model of the noise, namely a metric dynamical system $\left(\Omega, \mathcal{F}, \mathbb{P},\left(\theta_{t}\right)_{t \in \mathbb{T}}\right)$, where $(\Omega, \mathcal{F}, \mathbb{P})$ is a probability space and $(t, w) \mapsto \theta_{t} \omega$ is a measurable flow which leaves $\mathbb{P}$ invariant, i.e. $\theta_{t} \mathbb{P}=\mathbb{P}$ for all $t \in \mathbb{T}$.

(ii) A model of the system perturbed by noise, namely a cocycle $\varphi$ over $\theta$, i.e. a measurable mapping $\varphi: \mathbb{T} \times \Omega \times X \rightarrow X,(t, \omega, x) \mapsto \varphi(t, \omega, x)$, such that $(t, x) \mapsto \varphi(t, \omega, x)$ is continuous for all $\omega \in \Omega$ and the family $\varphi(t, \omega, \cdot)=\varphi(t, \omega): X \rightarrow X$ of random self-mappings of $X$ satisfies the cocycle property:

$$
\varphi(0, \omega)=\operatorname{id}_{X}, \varphi(t+s, \omega)=\varphi\left(t, \theta_{s} \omega\right) \circ \varphi(s, \omega) \quad \text { for all } \quad t, s \in \mathbb{T}, \omega \in \Omega .
$$

In this definition, $\mathbb{T}=\mathbb{Z}$ or $\mathbb{R}$.

It follows from (2.1) that $\varphi(t, \omega)$ is a homeomorphism of $X$, and the fact

$$
\varphi(t, \omega)^{-1}=\varphi\left(-t, \theta_{t} \omega\right)
$$

is very useful in the following.

Below any mapping from $\Omega$ into the collection of all subsets of $X$ is said to be a multifunction (or a set valued mapping) from $\Omega$ into X. We now give the definition of random set, which is a fundamental concept for RDS.

Definition 2.2 Let $X$ be a metric space with a metric d. The multifunction $\omega \mapsto D(\omega) \neq \emptyset$ is said to be a random set if the mapping $\omega \mapsto \operatorname{dist}_{X}(x, D(\omega))$ is measurable for any $x \in X$, where $\operatorname{dist}_{X}(x, B)$ is the distance in $X$ between the element $x$ and the set $B \subset X$. If $D(\omega)$ is closed/compact for each $\omega \in \Omega, D(\omega)$ is called a random closed/compact set.

Afterwards, we also call a multifunction $D(\omega)$ measurable for convenience if the mapping $\omega \mapsto \operatorname{dist}_{X}(x, D(\omega))$ is measurable for any $x \in X$.

Now we enumerate some basic results about random sets in the following propositions, for details the reader can refer to Arnold [1, Chueshov [4] for instance.

Proposition 2.1 Let $X$ be a Polish space, i.e. a separable complete metric space. The following assertions hold:

(i) $D$ is a random set in $X$ if and only if the set $\{\omega: D(\omega) \cap U \neq \emptyset\}$ is measurable for any open set $U \subset X$;

(ii) $D$ is a random set in $X$ if and only if $\overline{D(\omega)}$ is a random closed set $\overline{(D(\omega)}$ denotes the closure of $D(\omega)$ in $X)$;

(iii) if $D$ is a random open set, then the closure $\bar{D}$ of $D$ is a random closed set; if $D$ is a random closed set, then $\operatorname{int}(D)$, the interior of $D$, is a random open set;

(iv) $D$ is a random compact set in $X$ if and only if $D(\omega)$ is compact for every $\omega \in \Omega$ and the set $\{\omega: D(\omega) \cap C \neq \emptyset\}$ is measurable for any closed set $C \subset X$;

(v) if $\left\{D_{n}, n \in \mathbb{N}\right\}$ is a sequence of random closed sets with non-void intersection and there exists $n_{0} \in \mathbb{N}$ such that $D_{n_{0}}$ is a random compact set, then $\bigcap_{n \in \mathbb{N}} D_{n}$ is a random compact set in $X$;

(vi) if $\left\{D_{n}, n \in \mathbb{N}\right\}$ is a sequence of random sets, then $D=\bigcup_{n \in \mathbb{N}} D_{n}$ is also a random set in $X$; 
Proposition 2.2 (Measurable Selection Theorem). Let a multifunction $\omega \mapsto D(\omega)$ take values in the subspace of closed non-void subsets of a Polish space $X$. Then $D(\omega)$ is a random closed set if and only if there exists a sequence $\left\{v_{n}: n \in \mathbb{N}\right\}$ of measurable maps $v_{n}: \Omega \mapsto X$ such that

$$
v_{n}(\omega) \in D(\omega) \quad \text { and } \quad D(\omega)=\overline{\left\{v_{n}(\omega), n \in \mathbb{N}\right\}} \text { for all } \omega \in \Omega .
$$

In particular if $D(\omega)$ is a random closed set, then there exists a measurable selection, i.e. a measurable map $v: \Omega \mapsto X$ such that $v(\omega) \in D(\omega)$ for all $\omega \in \Omega$.

Similar to deterministic case, we can define random chain recurrence. The following random chain recurrent variable for random dynamical systems is the counterpart of chain recurrent point for the deterministic dynamical systems. Here, 'recurrence' is defined in the 'pull-back' sense.

Definition 2.3 For given random variable $\epsilon(\omega)>0$, the $n+1$ pairs $\left(x_{0}(\omega), t_{0}\right),\left(x_{1}(\omega), t_{1}\right)$, $\cdots,\left(x_{n}(\omega), t_{n}\right)$, where $x_{0}(\omega), x_{1}(\omega), \cdots, x_{n}(\omega)$ are random variables, are called a random $\epsilon(\omega)-T(\omega)$-chain, if the following holds:

$$
d\left(\varphi\left(t_{i}, \theta_{-t_{i}} \omega\right) x_{i}\left(\theta_{-t_{i}} \omega\right), x_{i+1}(\omega)\right)<\epsilon(\omega), \quad i=0, \cdots, n-1,
$$

where $t_{i} \geq T(\omega), T(\omega)$ is a positive random variable almost surely. And we call $n$ the length of the random $\epsilon(\omega)-T(\omega)$-chain. A random variable $x(\omega)$ is called random chain recurrent if for any given $\epsilon(\omega), T(\omega)>0$, there exists an $\epsilon(\omega)-T(\omega)$-chain beginning and ending at $x(\omega) \mathbb{P}$-almost surely; $x(\omega)$ is called partly random chain recurrent with index $\delta$ if for any $\epsilon(\omega), T(\omega)>0$, there exists an $\epsilon(\omega)-T(\omega)$-chain beginning and ending at $x(\omega)$ with probability not less than $\delta$, where the index $\delta$ is the maximal number satisfying this property; $x(\omega)$ is called completely random non-chain recurrent if there exists $\epsilon_{0}(\omega), T_{0}(\omega)>0$ such that there is no $\epsilon_{0}(\omega)-T_{0}(\omega)$-chain beginning and ending at $x(\omega)$ with positive probability.

Remark 2.1 In the definition of partly random chain recurrence, the index $\delta$ being maximal means that for $\forall \eta>0, \exists \epsilon_{0}(\omega), T_{0}(\omega)>0$ such that any $\epsilon_{0}(\omega)$ - $T_{0}(\omega)$-chain begins and ends at $x(\omega)$ with probability $\leq \delta+\eta$.

Remark 2.2 In this paper, we will denote $\mathcal{C R}_{\varphi}(\omega)$ the random chain recurrent set of $\varphi$, which has the property that for any random chain recurrent variable $x(\omega)$, we have $x(\omega) \in$ $\mathcal{C R}_{\varphi}(\omega) \mathbb{P}$-almost surely, and vice versa (i.e. if a random variable $x(\omega) \in \mathcal{C} \mathcal{R}_{\varphi}(\omega) \mathbb{P}$-almost surely, then $x(\omega)$ is random chain recurrent); for any completely random non-chain recurrent variable $x(\omega)$, we have $x(\omega) \in X-\mathcal{C R}_{\varphi}(\omega) \mathbb{P}$-almost surely, and vice versa; for any partly random chain recurrent variable $x(\omega)$ with index $\delta$, we have $x(\omega) \in \mathcal{C R}_{\varphi}(\omega)$ with probability $\delta$, and vice versa. For any given random variable $x(\omega)$, denote

$$
\Omega_{\mathcal{C R}}(x)=\left\{\omega \mid x(\omega) \in \mathcal{C R}_{\varphi}(\omega)\right\} .
$$

If $x(\omega)$ is a partly random chain recurrent variable with index $\delta$, then we call $\{x(\omega) \mid \omega \in$ $\left.\Omega_{\mathcal{C R}}(x)\right\}$ the chain recurrent part of $x(\omega)$. Therefore by the property of $\mathcal{C R}_{\varphi}(\omega)$, we have that $\mathcal{C R}_{\varphi}(\omega)$ is the union of all random chain recurrent variables and the chain recurrent part of those partly random chain recurrent variables.

Example 2.1 A simple example of random chain recurrent variable is random equilibrium (a random variable $x(\omega)$ is called an equilibrium if $\varphi(t, \omega) x(\omega)=x\left(\theta_{t} \omega\right)$ holds for $\forall t>$ $0, \omega \in \Omega)$.

Throughout the paper, we will assume that $X$ is a compact metric space, therefore it is a Polish space. The $\sigma$-algebra $\mathcal{F}^{u}$ of universally measurable sets associated with the base 
space $(\Omega, \mathcal{F})$ is defined by $\mathcal{F}^{u}=\bigcap_{\nu} \overline{\mathcal{F}}^{\nu}$, where the intersection is taken over all probability measures $\nu$ on $(\Omega, \mathcal{F})$ and $\overline{\mathcal{F}}^{\nu}$ denotes the completion of the $\sigma$-algebra $\mathcal{F}$ with respect to the measure $\nu$. And we call $\mathcal{F}^{u}$ the universal $\sigma$-algebra for brevity. For a random variable $T(\omega)$, we call $T(\omega)>0$ if it holds almost surely. By the measurable selection theorem, for any non-void random closed set, there exists random variables belonging to it. In the following, for a random open set, say $U(\omega)$, when we say that a random variable $x(\omega) \in U(\omega)$, we mean that there exists a random closed set $K(\omega) \subset U(\omega)$ such that $x(\omega) \in K(\omega)$ almost surely.

For late use, we give the following important projection theorem, which comes from $[3]$.

Proposition 2.3 (Projection Theorem). Let $X$ be a Polish space and $M \subset \Omega \times X$ be a set which is measurable with respect to the product $\sigma$-algebra $\mathcal{F} \times \mathcal{B}(X)$. Then the set

$$
\Pi_{\Omega} M=\{\omega \in \Omega:(\omega, x) \in M \text { for some } x \in X\}
$$

is universally measurable, i.e. belongs to $\mathcal{F}^{u}$, where $\Pi_{\Omega}$ stands for the canonical projection of $\Omega \times X$ to $\Omega$. In particular it is measurable with respect to the $\mathbb{P}$-completion $\overline{\mathcal{F}}^{\mathbb{P}}$ of $\mathcal{F}$.

Remark 2.3 We have the following direct result. If $M \in \mathcal{F}^{u} \times \mathcal{B}(X), \Pi_{\Omega} M \in \mathcal{F}^{u}$ too. In fact, we only need to show $\left(\mathcal{F}^{u}\right)^{u}=\mathcal{F}^{u}$ by projection theorem. To see this, we notice that, on the one hand, for arbitrary probability measure $\nu$ on measurable space $\left(\Omega, \mathcal{F}^{u}\right),\left.\nu\right|_{\mathcal{F}}$, the restriction of $\nu$ on $(\Omega, \mathcal{F})$, is a probability measure on $(\Omega, \mathcal{F})$. On the other hand, for arbitrary probability measure $\nu$ on $(\Omega, \mathcal{F})$, we can convert it into a probability measure on $\left(\Omega, \mathcal{F}^{u}\right)$ by adding subsets of $\Omega$ which are in $\mathcal{F}^{u}$ but not in $\mathcal{F}$ and defining their measures to be 0 . That is, the measures on $(\Omega, \mathcal{F})$ and those on $\left(\Omega, \mathcal{F}^{u}\right)$ are one to one. So by the fact that $\mathcal{F} \subset \mathcal{F}^{u} \subset \overline{\mathcal{F}}^{\nu}$, where $\nu$ is an arbitrary probability on $\left(\Omega, \mathcal{F}\right.$ or $\left.\mathcal{F}^{u}\right)$, we have

$$
\overline{\mathcal{F}}^{\nu} \subset(\overline{\mathcal{F}})^{\nu} \subset\left(\overline{\overline{\mathcal{F}}^{\nu}}\right)^{\nu}=\overline{\mathcal{F}}^{\nu} .
$$

Therefore

$$
\mathcal{F}^{u}=\bigcap_{\nu} \overline{\mathcal{F}}^{\nu} \subset \bigcap_{\nu}\left(\overline{\mathcal{F}^{u}}\right)^{\nu}=\left(\mathcal{F}^{u}\right)^{u} \subset \bigcap_{\nu} \overline{\mathcal{F}}^{\nu}=\mathcal{F}^{u},
$$

i.e. $\left(\mathcal{F}^{u}\right)^{u}=\mathcal{F}^{u}$.

By remark 2.3 without loss of generality, we need only consider $\mathcal{F}^{u}$-measurability throughout the paper.

At last, we give the definition of random local attractor and the random basin determined by it.

Definition 2.4 A random open set $U(\omega)$ is called random pre-attractor if it satisfies

$$
\overline{\bigcup_{t \geq T(\omega)} \varphi\left(t, \theta_{-t} \omega\right) U\left(\theta_{-t} \omega\right)} \subset U(\omega) \quad \text { for some } T(\omega)>0,
$$

where $T(\omega)$ is an $\mathcal{F}$-measurable random variable. And we define the random local attractor $A(\omega)$ inside $U(\omega)$ to be the following:

$$
A(\omega)=\bigcap_{n \in \mathbb{N}} \overline{\bigcup_{s \geq n T(\omega)} \varphi\left(s, \theta_{-s} \omega\right) U\left(\theta_{-s} \omega\right)} .
$$

And the random basin $B(A)(\omega)$ determined by $A(\omega)$ is defined as follows

$$
B(A)(\omega)=\left\{x: \varphi(t, \omega) x \in U\left(\theta_{t} \omega\right) \text { for some } t \geq 0\right\} .
$$


It is easy to see that in the above definition, the random basin $B(A)(\omega)$ may depend on the pre-attractor $U(\omega)$. In fact, we can show that the basin is independent of the choice of $U(\omega)$ and we defer the proof to the next section.

Example 2.2 Suppose $x(\omega)$ is a random variable, and $\epsilon_{0}(\omega), T_{0}(\omega)$ are two positive random variables. Consider the random set $U_{x}(\omega)$ determined by (3.11) in the next section. By the proof of lemma 3.7 we know that $U_{x}(\omega)$ is a random pre-attractor, but it is not necessarily a forward invariant random set. In fact, for $t<T_{0}(\omega)$, we can not obtain that (3.12) also holds, which guarantees that $U_{x}(\omega)$ is forward invariant. Hence the attractor $A_{x}(\omega)$ determined by $U_{x}(\omega)$ is not necessarily the attractor defined in [10.

\section{Proof of the main result}

Denote $U(T(\omega))=\overline{\bigcup_{s \geq T(\omega)} \varphi\left(s, \theta_{-s} \omega\right) U\left(\theta_{-s} \omega\right)}$.

Lemma 3.1 Suppose $U(\omega)$ is a given pre-attractor, then $U(T(\omega))$ and the the random local attractor

$$
A(\omega)=\bigcap_{n \in \mathbb{N}} U(n T(\omega))
$$

determined by $U(\omega)$ are random closed sets measurable with respect to $\mathcal{F}^{u}$. Moreover, $A(\omega)$ is invariant, i.e. $\varphi(t, \omega) A(\omega)=A\left(\theta_{t} \omega\right)$ for all $t \geq 0$, and $A(\omega)$ is a local random pull-back set attractor, therefore a local weak random set attractor in $U(\omega)$ (for the definition of weak random set attractor see [17]).

Proof. (i) We first show that $U(T(\omega))$ is a random closed set. The idea of the proof is borrowed from [1] and [4]. For every $x \in X$, define

$$
d(t, \omega):=\operatorname{dist}_{X}\left(x, \varphi\left(t, \theta_{-t} \omega\right) U\left(\theta_{-t} \omega\right)\right) .
$$

By (ii) of proposition 2.1 and the proof of proposition 1.5.1 of [4], we obtain that the function $(t, \omega) \mapsto d(t, \omega)$ is $\mathcal{B}(\mathbb{T}) \times \mathcal{F}$-measurable. Clearly we have

$$
\begin{gathered}
\operatorname{dist}_{X}(x, U(T(\omega)))=\operatorname{dist}_{X}\left(x, \bigcup_{t \geq T(\omega)} \varphi\left(t, \theta_{-t} \omega\right) U\left(\theta_{-t} \omega\right)\right) \\
=\inf _{t \geq T(\omega)} d(t, \omega) .
\end{gathered}
$$

For arbitrary $a \in \mathbb{R}^{+}$, we have

$$
\left\{\omega \mid \inf _{t \geq T(\omega)} d(t, \omega)<a\right\}=\Pi_{\Omega}\{(t, \omega) \mid d(t, \omega)<a, t \geq T(\omega)\} .
$$

It is obvious that the function $(t, \omega) \mapsto t-T(\omega)$ is measurable with respect to $\mathcal{B}(\mathbb{T}) \times \mathcal{F}$, so by projection theorem, we obtain that $\left\{\omega \mid \inf _{t \geq T(\omega)} d(t, \omega)<a\right\}$ is $\mathcal{F}^{u}$-measurable, which follows that $U(T(\omega))$ is a random set measurable with respect to the universal $\sigma$-algebra $\mathcal{F}^{u}$. The closeness of $U(T(\omega))$ is obvious.

(ii) Clearly we have $U(n T(\omega)) \supset U((n+1) T(\omega))$, which follows that

$$
A(\omega)=\lim _{n \rightarrow \infty} U(n T(\omega)) .
$$

Therefore $A(\omega)$ is a random closed set. To get the attraction property of $A(\omega)$, we notice that, for any given random compact set $K(\omega) \subset U(\omega)$,

$$
\lim _{t \rightarrow \infty} d\left(\varphi\left(t, \theta_{-t} \omega\right) K\left(\theta_{-t} \omega\right) \mid A(\omega)\right)=0
$$

holds almost surely by (3.4), where the metric $d(A \mid B)$ between two closed sets stands for the Hausdorff semi-metric, i.e. $d(A \mid B):=\sup _{x \in A} \inf _{y \in B} d(x, y)$. Hence $A(\omega)$ is a pull-back 
set attractor in $U(\omega)$. Then by the property of measure preserving of $\left\{\theta_{t}\right\}_{t \in \mathbb{T}}$, we obtain that

$$
\mathbb{P}-\lim _{t \rightarrow \infty} \mathrm{d}\left(\varphi(t, \omega) K(\omega), A\left(\theta_{t} \omega\right)\right)=0,
$$

which implies that $A(\omega)$ is a weak random set attractor in $U(\omega)$.

The rest work is to verify the invariance of $A(\omega)$. The forward invariance of $A(\omega)$ follows from [6], just changing a few details. For completeness, we give its proof here. For arbitrary $t \geq 0$,

$$
\begin{aligned}
& \varphi(t, \omega) A(\omega)=\varphi(t, \omega) \bigcap_{n \in \mathbb{N}} \overline{\bigcup_{\tau \geq n T(\omega)} \varphi\left(\tau, \theta_{-\tau} \omega\right) U\left(\theta_{-\tau} \omega\right)} \\
& \subset \bigcap_{n \in \mathbb{N}} \varphi(t, \omega) \overline{\bigcup_{\tau \geq n T(\omega)} \varphi\left(\tau, \theta_{-\tau} \omega\right) U\left(\theta_{-\tau} \omega\right)} \\
& \subset \bigcap_{n \in \mathbb{N}} \overline{\bigcup_{\tau \geq n T(\omega)} \varphi(t, \omega) \varphi\left(\tau, \theta_{-\tau} \omega\right) U\left(\theta_{-\tau} \omega\right)} \\
& =\bigcap_{n \in \mathbb{N}} \overline{\bigcup_{\tau \geq n T(\omega)} \varphi\left(t+\tau, \theta_{-(t+\tau)} \circ \theta_{t} \omega\right) U\left(\theta_{-(t+\tau)} \circ \theta_{t} \omega\right)} \\
& =\bigcap_{n \in \mathbb{N} \tau \geq n T(\omega)+t} \varphi\left(\tau, \theta_{-\tau} \circ \theta_{t} \omega\right) U\left(\theta_{-\tau} \circ \theta_{t} \omega\right) \\
& \subset \bigcap_{n \in \mathbb{N}} \overline{\bigcup_{\tau \geq n T(\omega)} \varphi\left(\tau, \theta_{-\tau} \circ \theta_{t} \omega\right) U\left(\theta_{-\tau} \circ \theta_{t} \omega\right)}=A\left(\theta_{t} \omega\right),
\end{aligned}
$$

where the first two inclusions follows from the facts $f\left(\bigcap_{\alpha} A_{\alpha}\right) \subset \bigcap_{\alpha} f\left(A_{\alpha}\right)$ for arbitrary $f$ and $f(\bar{A}) \subset \overline{f(A)}$ for $f$ continuous respectively. The backward invariance of $A(\omega)$ is similar to [1] noting that $X$ is compact, so we omit the details here. This completes the proof of the lemma.

Lemma 3.2 Suppose $A(\omega)$ is a random local attractor and $U_{1}(\omega), U_{2}(\omega)$ are two pre-attractors which determine the same attractor $A(\omega)$, then the two basins determined by $U_{1}, U_{2}$ respectively are equal $\mathbb{P}$-almost surely.

Proof. Denote $B_{1}(A)(\omega), B_{2}(A)(\omega)$ the basins determined by $U_{1}(\omega), U_{2}(\omega)$ respectively. For arbitrary random variable $x(\omega) \in B_{1}(A)(\omega)$, there exists $t(\omega) \geq 0$ such that

$$
\varphi(t(\omega), \omega) x(\omega) \in U_{1}\left(\theta_{t(\omega)} \omega\right), \forall \omega \in \Omega
$$

by the definition of basin. By attraction property of $A(\omega)$ and the measure preserving of $\theta_{t}$, it follows that for $\forall \epsilon>0$, the following holds:

$$
\begin{aligned}
& \lim _{s \rightarrow \infty} \mathbb{P}\left\{\omega \mid \operatorname{dist}_{X}\left(\varphi\left(s, \theta_{t(\omega)} \omega\right) \varphi(t(\omega), \omega) x(\omega), A\left(\theta_{s} \circ \theta_{t(\omega)} \omega\right)\right)>\epsilon\right\} \\
= & \lim _{s \rightarrow \infty} \mathbb{P}\left\{\omega \mid \operatorname{dist}_{X}\left(\varphi(s+t(\omega), \omega) x(\omega), A\left(\theta_{s+t(\omega)} \omega\right)\right)>\epsilon\right\} \\
= & \lim _{s \rightarrow \infty} \mathbb{P}\left\{\omega \mid \operatorname{dist}_{X}\left(\varphi(s, \omega) x(\omega), A\left(\theta_{s} \omega\right)\right)>\epsilon\right\} \\
= & \lim _{s \rightarrow \infty} \mathbb{P}\left\{\omega \mid \operatorname{dist}_{X}\left(\varphi\left(s, \theta_{-s} \omega\right) x\left(\theta_{-s} \omega\right), A(\omega)\right)>\epsilon\right\}=0 .
\end{aligned}
$$

Denote $d(\omega):=\operatorname{dist}_{X}\left(A(\omega), X-U_{2}(\omega)\right)$, where the distance $\operatorname{dist}_{X}(A, B)$ between two closed sets stands for the smallest distance between them, i.e. $\operatorname{dist}_{X}(A, B)=\inf _{x \in A} \inf _{y \in B} d(x, y)$. Therefore we have $d(\omega)>0$ almost surely by the compactness of $X$. By a standard argument, we obtain that there exists a $\delta>0$ such that

$$
\mathbb{P}\{\omega \mid d(\omega)>\delta\} \geq 1-\epsilon .
$$


Denote $\Omega_{\delta}:=\{\omega \mid d(\omega)>\delta\}$. By 3.5 wave

$$
\lim _{s \rightarrow \infty} \mathbb{P}\left\{\omega \in \Omega_{\delta} \mid d\left(\varphi\left(s, \theta_{-s} \omega\right) x\left(\theta_{-s} \omega\right), A(\omega)\right)>\frac{\delta}{4}\right\}=0 .
$$

Therefore there exists $t_{\delta}(\omega) \geq 0$ such that

$$
d\left(\varphi\left(s, \theta_{-s} \omega\right) x\left(\theta_{-s} \omega\right), A(\omega)\right)<\frac{\delta}{2}, s \geq t_{\delta}(\omega)
$$

holds almost surely on $\Omega_{\delta}$. Hence by the definition of $B_{2}(A)(\omega)$ and the measure preserving of $\theta_{t}$, we get that

$$
\begin{gathered}
\mathbb{P}\left\{\omega \mid x(\omega) \in B_{2}(A)(\omega)\right\}=\mathbb{P}\left\{\omega \mid \varphi\left(s, \theta_{-s} \omega\right) x\left(\theta_{-s} \omega\right) \in U_{2}(\omega) \text { for some } s \geq 0\right\} \\
\geq \mathbb{P}\left(\Omega_{\delta}\right) \geq 1-\epsilon .
\end{gathered}
$$

Since $\epsilon>0$ is arbitrary, we have $x(\omega) \in B_{2}(A)(\omega)$ almost surely by letting $\epsilon \rightarrow 0$. It follows that $B_{1}(A)(\omega) \subset B_{2}(A)(\omega)$ almost surely, and the converse inclusion is similar. This terminates the proof of the lemma.

The above lemma indicates that the basin $B(A)(\omega)$ is uniquely determined by $A(\omega)$, therefore is well defined. The following lemma says that the basin is backward invariant random open.

Lemma 3.3 For any given random local attractor $A(\omega)$, the random basin $B(A)(\omega)$ determined by $A(\omega)$ is a backward invariant random open set.

Proof. It is obvious that $x \in B(A)(\omega)$ is equivalent to $\varphi(t, \omega) x \in U\left(\theta_{t} \omega\right)$ for some $t \geq 0$, i.e. $x \in \varphi\left(-t, \theta_{t} \omega\right) U\left(\theta_{t} \omega\right)$ by using the fact that $\varphi(t, \omega)^{-1}=\varphi\left(-t, \theta_{t} \omega\right)$. So it follows that $x \in B(A)(\omega)$ if and only if $x \in \bigcup_{t \geq 0} \varphi\left(-t, \theta_{t} \omega\right) U\left(\theta_{t} \omega\right)$, therefore we obtain that

$$
B(A)(\omega)=\bigcup_{t \geq 0} \varphi\left(-t, \theta_{t} \omega\right) U\left(\theta_{t} \omega\right) .
$$

Then by a similar method to that of [1] 6, 4, we can easily obtain that

$$
\overline{\bigcup_{t \geq 0} \varphi\left(-t, \theta_{t} \omega\right) \overline{U\left(\theta_{t} \omega\right)}}
$$

is $\mathcal{F}^{u}$-measurable, hence it is a random closed set. It is easy to verify that we have the following holds

$$
\overline{\bigcup_{t \geq 0} \varphi\left(-t, \theta_{t} \omega\right) U\left(\theta_{t} \omega\right)}=\overline{\bigcup_{t \geq 0} \varphi\left(-t, \theta_{t} \omega\right) \overline{U\left(\theta_{t} \omega\right)}}
$$

To see this, note first that the left hand is obvious the subset of the right hand. And every element of the union of right hand is a subset of the left hand, so the closure of the union of right is included by the left for the closeness of the left hand. So we have got that the closure of $B(A)(\omega)$ is a random closed set. By (ii) of proposition 2.1 we obtain that $B(A)(\omega)$ is a random set, the openness of $B(A)(\omega)$ follows the fact that $\varphi\left(-t, \theta_{t} \omega\right)$ is homeomorphism on $X$.

To show the backward invariance of $B(A)(\omega)$, we only need to show that its complement $X-B(A)(\omega)$ is forward invariant. If the assertion is false, then there exists an $x_{0} \in$ $X-B(A)(\omega)$ and $t_{0}>0$ such that $\varphi\left(t_{0}, \omega\right) x_{0} \in B(A)\left(\theta_{t_{0}} \omega\right)$. Then by the definition of random basin, we have $\varphi\left(t_{1}, \theta_{t_{0}} \omega\right) \varphi\left(t_{0}, \omega\right) x_{0} \in U\left(\theta_{t_{0}+t_{1}} \omega\right)$ for some $t_{1} \geq 0$, where $U$ is a random pre-attractor which determines $A$. But by the definition of random basin, $X-B(A)(\omega)$ is the set of points that never enter $U$, a contradiction. This terminates the proof of the lemma.

By the above preliminaries, we can prove our main theorem now. We decompose the proof of the main theorem into the following several lemmas: 
Lemma 3.4 If the random chain recurrent variable $x(\omega) \in U(\omega) \mathbb{P}$-almost surely, where $U(\omega)$ is a random pre-attractor, then we have $x(\omega) \in A(\omega) \mathbb{P}$-almost surely, where $A(\omega)$ is the attractor determined by $U(\omega)$.

Proof. If $x(\omega) \in U(\omega) \mathbb{P}$-almost surely, recalling that $U(\omega)$ is a random pre-attractor, fix $T(\omega)>0$ such that $U(T(\omega)) \subset U(\omega)$. Denote

$$
\bar{d}(\omega):=\operatorname{dist}_{X}(U(T(\omega)), X-U(\omega)) .
$$

By the compactness of $X$, it is obvious that $\bar{d}(\omega)>0$ holds almost surely. By measurable selection theorem, similar to [2, there exists two collections of random variables

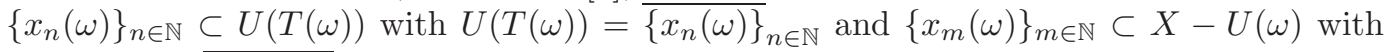
$X-U(\omega)={\overline{\left\{x_{m}(\omega)\right\}_{m \in \mathbb{N}}}}_{\text {such that }}$

$$
\bar{d}(\omega)=\inf _{n \in \mathbb{N}, m \in \mathbb{N}} d\left(x_{n}(\omega), x_{m}(\omega)\right) .
$$

So we obtain that $\bar{d}(\omega)$ is a random variable. Choose $0<\epsilon(\omega)<\bar{d}(\omega)$, then we have

$$
\varphi\left(t, \theta_{-t} \omega\right) x\left(\theta_{-t} \omega\right) \in \varphi\left(t, \theta_{-t} \omega\right) U\left(\theta_{-t} \omega\right) \subset U(T(\omega)), \text { where } t \geq T(\omega) .
$$

Then by the the fact that $x(\omega)$ is random chain recurrent, for this $\epsilon(\omega)>0$ and the above $T(\omega)$, there exists an $\epsilon(\omega)$-T( $\omega)$-chain $\left(x_{0}(\omega), t_{0}\right),\left(x_{1}(\omega), t_{1}\right), \cdots,\left(x_{n}(\omega), t_{n}\right)$ with $x_{0}(\omega)=x_{n}(\omega)=x(\omega)$. So by the choice of $\epsilon(\omega)$ and induction we obtain that

$$
x(\omega) \in B_{\epsilon(\omega)}(U(T(\omega))) \subset U(\omega)
$$

almost surely, where $B_{r}(x)$ stands for the open ball centered at $x$ with radius $r$.

Hence we derive $x(\omega) \in U(T(\omega))$ from letting $\epsilon(\omega) \rightarrow 0$ in (3.9) and from the closeness of $U(T(\omega))$. And then let $T(\omega) \rightarrow \infty$ in (3.9), we obtain $x(\omega) \in A(\omega)$ almost surely by (3.4).

Lemma 3.5 Suppose $U(\omega)$ is a random open set, $x(\omega)$ is a random variable. Define

$$
t(\omega):=\inf \left\{t \in \mathbb{R}^{+} \mid \varphi(t, \omega) x(\omega) \in \overline{U\left(\theta_{t} \omega\right)}\right\},
$$

i.e. the first entrance time of $x(\omega)$ into $U(\omega)$ under the cocycle $\varphi$. Then $t(\omega)$ is a random variable, which is measurable with respect to the universal $\sigma$-algebra $\mathcal{F}^{u}$.

Proof. It is easy to see that

$$
t(\omega)=\inf \left\{t \in \mathbb{R}^{+} \mid \operatorname{dist}_{X}\left(\varphi(t, \omega) x(\omega), \overline{U\left(\theta_{t} \omega\right)}\right)=0\right\} .
$$

Since the function

$$
(t, \omega) \mapsto \operatorname{dist}_{X}\left(\varphi(t, \omega) x(\omega), \overline{U\left(\theta_{t} \omega\right)}\right)=: \tilde{d}(t, \omega)
$$

is $\mathcal{B}(\mathbb{T}) \times \mathcal{F}$-measurable by a similar argument as the proof of lemma 3.1 For arbitrary $a \in \mathbb{R}^{+}$, it is easy to see that

$$
\{\omega \mid t(\omega)<a\}=\Pi_{\Omega}\{(t, \omega) \mid 0 \leq t<a, \tilde{d}(t, \omega)=0\} .
$$

It is obvious that $\{(t, \omega) \mid 0 \leq t<a, \tilde{d}(t, \omega)=0\} \in \mathcal{B}(\mathbb{T}) \times \mathcal{F}$, so $\{\omega \mid t(\omega)<a\}$ is $\mathcal{F}^{u}$ measurable by projection theorem.

Lemma 3.6 If the random chain recurrent variable $x(\omega) \in B(A)(\omega) \mathbb{P}$-almost surely, then we have $x(\omega) \in A(\omega) \mathbb{P}$-almost surely, where $B(A)(\omega)$ is the basin determined by the random local attractor $A(\omega)$. 
Proof. For $x(\omega) \in B(A)(\omega)$, take $t(\omega) \geq 0$ defined by (3.10). Suppose $U(\omega)$ is a preattractor which determines the attractor $A(\omega)$. So we have, for $s \geq T\left(\theta_{t(\omega)} \omega\right)$, the following almost surely holds:

$$
\begin{aligned}
\varphi(s+t(\omega), \omega) x(\omega) & =\varphi\left(s, \theta_{t(\omega)} \omega\right) \varphi(t(\omega), \omega) x(\omega) \\
& \in \overline{\varphi\left(s, \theta_{t(\omega)} \omega\right) U\left(\theta_{t(\omega)} \omega\right)} \subset^{*} U\left(\theta_{s+t(\omega)} \omega\right) .
\end{aligned}
$$

In fact, by the definition of pre-attractor, there exists a random variable $T\left(\theta_{t(\omega)} \omega\right)>0$ such that

$$
\overline{\bigcup_{s \geq T\left(\theta_{t(\omega)} \omega\right)} \varphi\left(s, \theta_{-s} \circ \theta_{t(\omega)} \omega\right) U\left(\theta_{-s} \circ \theta_{t(\omega)} \omega\right)} \subset U\left(\theta_{t(\omega)} \omega\right) .
$$

Then by the property of measure preserving of $\theta_{t}$, we obtain that $\subset^{*}$ holds $\mathbb{P}$-almost surely. Hence we obtain

$$
\begin{aligned}
& \mathbb{P}\left\{\omega \mid \varphi(s+t(\omega), \omega) x(\omega) \in U\left(\theta_{s+t(\omega)} \omega\right), s \geq T\left(\theta_{t(\omega)} \omega\right)\right\} \\
= & \mathbb{P}\left\{\omega \mid \varphi\left(s+t(\omega), \theta_{-(s+t(\omega))} \omega\right) x\left(\theta_{-(s+t(\omega))} \omega\right) \in U(\omega), s \geq T\left(\theta_{t(\omega)} \omega\right)\right\} \\
= & 1
\end{aligned}
$$

by the fact that $\mathbb{P}$ is invariant under $\theta_{t}$ again. Further more, we are able to obtain the following finer result:

$$
\begin{aligned}
& \varphi\left(s+t(\omega), \theta_{-(s+t(\omega))} \omega\right) x\left(\theta_{-(s+t(\omega))} \omega\right) \\
= & \varphi\left(s, \theta_{-s} \omega\right) \varphi\left(t(\omega), \theta_{-(s+t(\omega))} \omega\right) x\left(\theta_{-(s+t(\omega))} \omega\right) \\
\epsilon^{*} & \overline{\varphi\left(s, \theta_{-s} \omega\right) U\left(\theta_{-s} \omega\right)} \subset^{* *} U(\omega),
\end{aligned}
$$

where $\epsilon^{*}$ holds using the property of measure preserving of $\theta_{t}$ again. And $\subset^{* *}$ holds if $s \geq T(\omega)$ by the property of pre-attractor. So denote $\tilde{T}(\omega)=\max \left\{T(\omega), T\left(\theta_{t(\omega)} \omega\right)\right\}$, and take $T_{1}(\omega)=t(\omega)+\tilde{T}(\omega)$, then it follows that

$$
\overline{\varphi\left(s, \theta_{-s} \omega\right) U\left(\theta_{-s} \omega\right)} \subset U(T(\omega)) \subset U(\omega)
$$

whenever $s \geq T_{1}(\omega)$. Take $\bar{d}(\omega)$ as defined by (3.7), fix $T_{1}(\omega)$, choose $0<\epsilon(\omega)<d(\omega)$. Then it follows that any random $\epsilon(\omega)-T_{1}(\omega)$-chain of length one begins at $x(\omega) \in B(A)(\omega)$ must ends in $B_{\epsilon(\omega)}(U(T(\omega)))$ almost surely. By the fact that $x(\omega)$ is a random chain recurrent variable and repeat the proof process of lemma 3.4 we obtain that $x(\omega) \in A(\omega)$ almost surely.

Remark 3.1 By the process of proofs of lemma 3.4 and lemma 3.6] if

$$
\mathbb{P}\{\omega \mid x(\omega) \in B(A)(\omega)\}=\delta<1 \text {, and } x(\omega) \text { is random chain recurrent, }
$$

then we can easily obtain that

$$
\mathbb{P}\{\omega \mid x(\omega) \in A(\omega)\}=\delta,
$$

i.e.

$$
\mathbb{P}\{\omega \mid x(\omega) \in B(A)(\omega) \backslash A(\omega)\}=0 .
$$

If $x(\omega)$ is partly random chain recurrent with index $\delta$, then we have if $\omega \in \Omega_{\mathcal{C R}}(x)$, then

$$
x(\omega) \in A(\omega) \text { or } x(\omega) \in X \backslash B(A)(\omega)
$$


holds except for a null set for any given attractor $A(\omega)$ by the proof process of lemma 3.4 and lemma 3.6 again. Hence we obtain that

$$
\omega \in \Omega \backslash \Omega_{\mathcal{C R}}(x) \text { whenever } x(\omega) \in B(A)(\omega)-A(\omega),
$$

i.e.

$$
x(\omega) \in X-\mathcal{C R}_{\varphi}(\omega) \text { whenever } x(\omega) \in B(A)(\omega)-A(\omega) .
$$

Therefore by lemma 3.4 lemma 3.6 and above arguments we obtain that

$$
B(A)(\omega)-A(\omega) \subset X-\mathcal{C} \mathcal{R}_{\varphi}(\omega)
$$

holds $\mathbb{P}$-almost surely for any given attractor $A(\omega)$. When the number of attractors is uncountable, to avoid the possibility that

$$
\bigcup[B(A)(\omega)-A(\omega)] \subset X-\mathcal{C} \mathcal{R}_{\varphi}(\omega)
$$

does not hold $\mathbb{P}$-almost surely, we can redefine $B(A)(\omega)-A(\omega)$ on the null set such that

$$
B(A)(\omega)-A(\omega) \subset X-\mathcal{C R}_{\varphi}(\omega) \text { for all } \omega \in \Omega .
$$

In fact, if $B(A)(\omega)-A(\omega) \subset X-\mathcal{C R}_{\varphi}(\omega)$ holds on a set $\tilde{\Omega}$ of full measure, we can set

$$
B(A)(\omega)-A(\omega)=X-\mathcal{C} \mathcal{R}_{\varphi}(\omega) \text { on } \Omega \backslash \tilde{\Omega} .
$$

Hence by this way, we have obtained

$$
\bigcup[B(A)(\omega)-A(\omega)] \subset X-\mathcal{C} \mathcal{R}_{\varphi}(\omega)
$$

holds $\mathbb{P}$-almost surely.

By lemma 3.4 lemma 3.6 and remark 3.1 we obtain that the right hand of [1.4 is a subset of the left hand $\mathbb{P}$-almost surely. To prove the equality (1.4), the rest work is to verify that the converse inclusion is true $\mathbb{P}$-almost surely. To this end, we first define a random open set $U_{x}(\omega)$ measurable with respect to $\mathcal{F}^{u}$ for late use, which is defined as follows.

Suppose $x(\omega)$ is a random variable, $\epsilon_{0}(\omega), T_{0}(\omega)$ are two positive random variables. Define

$$
\begin{array}{cc}
U_{1}(\omega): & =\bigcup_{t \geq T_{0}(\omega)} B_{\epsilon_{0}(\omega)}\left(\varphi\left(t, \theta_{-t} \omega\right) x\left(\theta_{-t} \omega\right)\right), \\
U_{2}(\omega):=\bigcup_{t \geq T_{0}(\omega)} B_{\epsilon_{0}(\omega)}\left(\varphi\left(t, \theta_{-t} \omega\right) U_{1}\left(\theta_{-t} \omega\right)\right), & \ldots \ldots \ldots \ldots \\
& \ldots \ldots \ldots \ldots \\
U_{n}(\omega):=\bigcup_{t \geq T_{0}(\omega)} B_{\epsilon_{0}(\omega)}\left(\varphi\left(t, \theta_{-t} \omega\right) U_{n-1}\left(\theta_{-t} \omega\right)\right),
\end{array}
$$

By the proof method of proposition 1.5.1 of [4] on page 32, which in turn stems from [11, and a similar argument as in lemma 3.3 adding some slight changes in the process of proof, we can conclude that $U_{1}(\omega), \cdots, U_{n}(\omega), \cdots$ are all $\mathcal{F}^{u}$-measurable open sets. We omit the details here. So the set

$$
U_{x}(\omega):=\bigcup_{n \in \mathbb{N}} U_{n}(\omega)
$$

is a random open set measurable with respect to $\mathcal{F}^{u}$ by (vi) of proposition 2.1.

Now we can give the proof of the converse inclusion of (1.4), see the following lemma. 
Lemma 3.7 $X-\mathcal{C R}_{\varphi}(\omega) \subset \bigcup[B(A)(\omega)-A(\omega)]$ holds $\mathbb{P}$-almost surely.

Proof. We divide the proof into two cases.

Case 1: when $x(\omega)$ is completely random non-chain recurrent, i.e. $x(\omega) \in X-\mathcal{C R}_{\varphi}(\omega)$ $\mathbb{P}$-almost surely, there exists $\epsilon_{0}(\omega)>0, T_{0}(\omega)>0$ such that there is no $\epsilon_{0}(\omega)-T_{0}(\omega)$-chain which begins and ends at $x(\omega)$ with positive probability. Take $U_{x}(\omega)$ defined by (3.11), then it is easy to see that $x(\omega) \notin U_{x}(\omega)$ and

$$
\varphi\left(t, \theta_{-t} \omega\right) x\left(\theta_{-t} \omega\right) \in U_{x}(\omega)
$$

when $t \geq T_{0}(\omega)$. Given $y(\omega) \in U_{x}(\omega)$, it is obvious that the $\epsilon_{0}(\omega)$-neighbourhood of $\varphi\left(t, \theta_{-t} \omega\right) y\left(\theta_{-t} \omega\right)$ lies in $U_{x}(\omega)$ whenever $t \geq T_{0}(\omega)$, therefore $U_{x}\left(T_{0}(\omega)\right) \subset U_{x}(\omega)$, where $U_{x}\left(T_{0}(\omega)\right)$ is defined similarly to $U(T(\omega))$. So $U_{x}(\omega)$ is a random pre-attractor and it determines a random local attractor $A_{x}(\omega)$ by lemma 3.1 (The only difference is that $U_{x}(\omega)$ is $\mathcal{F}^{u}$ measurable while the $U(\omega)$ in lemma 3.1 is $\mathcal{F}$-measurable. But by remark 2.3 we know that this does not affect the result). And we have $x(\omega) \in B\left(A_{x}\right)(\omega)-U_{x}(\omega) \subset B\left(A_{x}\right)(\omega)-A_{x}(\omega)$ by (3.12).

Case 2: when $x(\omega)$ is partly random chain recurrent with index $\delta$, i.e. $x(\omega) \in X-\mathcal{C R}_{\varphi}(\omega)$ with probability $1-\delta$, then for $\forall \eta>0, \exists \epsilon_{0}(\omega), T_{0}(\omega)>0$ such that any $\epsilon_{0}(\omega)-T_{0}(\omega)$-chain begins and ends at $x(\omega)$ with probability $\leq \delta+\eta$. Take $U_{x}(\omega)$ defined by (3.11), then by the proof of case 1 , it is easy to see that $x(\omega) \in B\left(A_{x}\right)(\omega) \mathbb{P}$-almost surely and

$$
\mathbb{P}\left\{\omega \mid x(\omega) \in U_{x}(\omega)\right\} \leq \delta+\eta,
$$

hence

$$
\mathbb{P}\left\{\omega \mid x(\omega) \in B\left(A_{x}\right)(\omega)-A_{x}(\omega)\right\} \geq 1-(\delta+\eta) .
$$

Thus we obtain

$$
\mathbb{P}\{\omega \mid x(\omega) \in \bigcup[B(A)(\omega)-A(\omega)]\} \geq 1-\delta
$$

by letting $\eta \rightarrow 0$.

Therefore, by the proof of case 1 and case 2 , we obtain

$$
X-\mathcal{C R}_{\varphi}(\omega) \subset \bigcup[B(A)(\omega)-A(\omega)]
$$

holds $\mathbb{P}$-almost surely. This completes the proof of the lemma.

By lemmas 3.4 3.6 and 3.7 and remark 3.1 we complete the proof of our main theorem.

\section{Two simple examples}

In this section, we will give two examples to illustrate our results. The first example is borrowed from [10].

Example 4.1 Consider the Stratonovich stochastic differential equation (SDE)

$$
\mathrm{d} X_{t}=\left(X_{t}-X_{t}^{3}\right) \mathrm{d} t+\left(X_{t}-X_{t}^{3}\right) \circ \mathrm{d} W_{t}
$$

on the interval $[-1,1]$. From p.123 of [16] we know that the $\operatorname{RDS} \varphi: \mathbb{R} \times \Omega \times[-1,1] \mapsto[-1,1]$ generated by this SDE can be expressed by

$$
\varphi(t, \omega) x=\frac{x \mathrm{e}^{t+W_{t}(\omega)}}{\left(1-x^{2}+x^{2} \mathrm{e}^{2 t+2 W_{t}(\omega)}\right)^{\frac{1}{2}}} .
$$


It is easily to see that $A_{1}=\{-1\}, A_{2}=\{1\}, A_{3}=\{-1,1\}$ are all attractors except for the two trivial attractors $\emptyset,[-1,1]$, and the corresponding basins of attraction are $[-1,0),(0,1]$ and $[-1,0) \cup(0,1]$ respectively. Hence by our main theorem, we obtain that the random chain recurrent variables are $x_{1}(\omega) \equiv-1, x_{2}(\omega) \equiv 1$ and $x_{3}(\omega) \equiv 0$ and the combinations of $-1,0,1$, e.g. $x(\omega)=-1,0,1$ with probabilities $\frac{1}{4}, \frac{1}{4}, \frac{1}{2}$ respectively; all random variables taking values in $(-1,0) \bigcup(0,1)$ are completely random non-chain recurrent variables; and the random variables taking values $1,-1,0$ with positive probability and also taking values in $(-1,0) \bigcup(0,1)$ with positive probability are partly random chain recurrent variables.

Example 4.2 Let the probability space $(\Omega, \mathcal{F}, \mathbb{P})$ be given by $\Omega=S^{1}$ with $\mathcal{F}=\mathcal{B}(\Omega)$, and $\mathbb{P}$ the Lebesgue measure. Put $\theta_{t} \omega=\omega+t$. Let the state space $X=S^{1}$, too. Define a random homeomorphism $\psi(\omega): S^{1} \rightarrow S^{1}$ by $\psi(\omega) x=x+\omega$. We define an RDS by

$$
\varphi(t, \omega)=\psi\left(\theta_{t} \omega\right) \circ \varphi_{0}(t) \circ \psi^{-1}(\omega),
$$

where $\varphi_{0}$ is the flow on $S^{1}$ determined by the equation

$$
\dot{x}=-\cos x .
$$

Then the $\operatorname{RDS} \varphi$ has no non-trivial attractor besides $X, \emptyset$, for the details see 8 . Hence by our main theorem, the random chain recurrent set is $X$, i.e. all random variables are random chain recurrent.

\section{Some discussions}

We know very well that there are several nonequivalent definitions of random attractors for random dynamical systems, see [18 for instance. Pull-back attractors were introduced and studied by Crauel and Flandoli [11, Crauel, Debussche, and Flandoli [9, Schmalfuss [19, 20] and others. Ochs in [17] firstly introduced random weak attractors, where 'weak' means that the convergence to attractor is in probability instead of usual almost sureness. Another kind of attractor is forward attractor, which is in contrast to pull-back attractor and whose convergence is almost sure convergence in contrast to weak attractor's convergence in probability. As stated in 2, the choice of convergence in probability makes the forward and pull-back attractors equivalent. So the authors adopted the weak attraction, in fact they adopt the weak point attractor, in order to prove Lyapunov's second method for RDS, for details see [2. In order to get the Morse theory for RDS, the authors in 10 introduced the definition of attractor-repeller pair for RDS. They defined an attractor to be the maximal invariant random compact set inside its fundamental neighbourhood, i.e. a forward invariant random open set, and defined the repeller corresponding to it to be the complement of the basin of the attractor. For details, see [10].

In this paper, we adopt the definition 2.4 Now we simply discuss the relations between it and the old ones. It is obvious that, for a random pre-attractor $U(\omega)$, the random attractor $A(\omega)$ determined by it in definition 2.4 is a random pull-back attractor in the universe

$$
\mathcal{D}=\{D(\omega) \mid \overline{D(\omega)} \subset U(\omega) \text { and } D(\omega) \text { is measurable }\},
$$

therefore it is a weak set attractor, and therefore a weak point attractor in $\mathcal{D}$. But it is obvious not necessarily a forward attractor in $\mathcal{D}$. As mentioned above, the authors in [10] introduced the definition of attractor-repeller pair for RDS. It seems that their definition of attractor works for our purpose, too. But when we prove lemma 3.7 we do not know how to construct a fundamental neighbourhood $U(\omega)$ defined in [10 to obtain the attractor. With respect to the relations between our attractor and the attractor of [10, the difference is that in our definition, the random pre-attractor is not necessarily forward invariant, while the 
fundamental neighbourhood in [10] is forward invariant; our attractor is pull-back attractor in the universe $\mathcal{D}$ defined above, while the attractor in [10] is only a weak set attractor inside its fundamental neighbourhood. Hence our attractor is not weaker than the attractor in [10, and vice versa.

In fact, besides these, our definition of attractor approximates closely to Conley's deterministic definition of attractor. Conley in [5] defined an attractor to be an invariant compact set which is the omega-limit set of one of its neighbourhoods. A random attractor defined by definition 2.4 is obvious the omega-limit set ( a random set $A(\omega)$ is called the omega-limit set of $D(\omega)$ if $\left.A(\omega)=\lim _{t \rightarrow \infty} \varphi\left(t, \theta_{-t} \omega\right) D\left(\theta_{-t} \omega\right)\right)$ of one of the pre-attractors determining it. Conversely, if an invariant random compact set is the omega-limit set of one of its neighbourhoods, then, by the definition of omega-limit set, we can see that the neighbourhood must satisfies (2.2), except that the $T(\omega)$ in (2.2) is not necessarily measurable, which guarantees the measurability of $U(T(\omega)), A(\omega)$ etc.

In this paper we adopt the form

$$
X-\mathcal{C R}(\varphi)=\bigcup[B(A)-A]
$$

instead of the original form

$$
\mathcal{C R}(\varphi)=\bigcap[A \cup R]
$$

in our main theorem, where $R$ denotes the repeller corresponding to the attractor $A$. On the one hand the form we employ here is also adopted by other authors, see 13, 14, 15] for instances. In fact we are inspired by these references. On the other hand, the random pre-attractor is not necessarily forward invariant, which restricts us to obtain the invariance of the basin of attractor as in [10. Hence we can not obtain the invariance of $X-B(A)(\omega)$, but it is forward invariant, see lemma 3.3 So we can not define $X-B(A)(\omega)$ to be the repeller corresponding to the attractor $A(\omega)$ as in [10, for a repeller should be invariant.

With respect to the measurability, we find it not appropriate to confine us to considering $\mathcal{F}$-measurability only. Since it is easy to see that $U(T(\omega)), B(A)(\omega), A(\omega), d(\omega)$ etc in this paper are not measurable with respect to $\mathcal{F}$. Therefore to serve our purpose, we have to allow measurability with respect to other $\sigma$-algebra, in fact we allow $\mathcal{F}^{u}$-measurability. This treatment is also adopted by others, see 6, 7, 9, 11, 4, for instance. Certainly we can choose $\overline{\mathcal{F}}^{\mathbb{P}}$, the $\mathbb{P}$-completion $\sigma$-algebra of $\mathcal{F}$, in order that the above variables are measurable. Of course, when $\mathbb{T}=\mathbb{Z}$, i.e. the RDS in consideration is discrete, or $\mathcal{F}$ is complete with respect to the probability measure $\mathbb{P}$ on base space $(\Omega, \mathcal{F})$, i.e. $\mathcal{F}=\overline{\mathcal{F}}^{\mathbb{P}}$, all the random variables are measurable with respect to $\mathcal{F}$ as usual. Whence in this case, only considering $\mathcal{F}$-measurability as usual is enough to obtain our results.

\section{Acknowledgements}

The author expresses his sincere thanks to Professor Yong Li for his instructions and many invaluable suggestions. The author is very grateful to Professor Youqing Ji and Qingdao Huang for helpful discussions. The author is also grateful to the anonymous referees for their careful reading the manuscript and helpful suggestions, one of whom has pointed out an important oversight and some confusing notations.

\section{References}

[1] Arnold L 1998 Random Dynamical Systems (Berlin Heidelberg New York: SpringerVerlag)

[2] Arnold L and Schmalfuss B 2001 Lyapunovs Second Method for Random Dynamical Systems J. Diff. Equ. 177 235-65 
[3] Castaing C and Valadier M 1977 Convex Analysis and Measurable Multifunctions (Lec. Notes in Math. vol 580) (Berlin: Springer-Verlag)

[4] Chueshov I 2002 Monotone Random systems Theory and Applications (Lec. Notes in Math. vol 1779) (Berlin Heidelberg: Springer-Verlag)

[5] Conley C 1978 Isolated Invariant Sets and the Morse Index (Conf. Board Math. Sci. vol 38) (Providence: Amer. Math. Soc.)

[6] Crauel H 1999 Global random attractors are uniquely determined by attracting deterministic compact sets Ann. Mat. Pura Appl. (IV) 176 57-72

[7] Crauel H 2001 Random Point Attractors versus Random Set Attractors J. London Math. Society 63 (2) 413-27

[8] Crauel H 2002 A uniformly exponential random forward attractor which is not a pullback attractor Arch. Math. 78 329-36

[9] Crauel H, Debussche A and Flandoli F 1997 Random Attractors J. Dyn. Diff. Equ. 9 $307-41$

[10] Crauel H, Duc L H and Siegmund S 2004 Towards a Morse heory for random dyanamical systems Stochastics and Dynamics 4 (3) 277-96

[11] Crauel H and Flandoli F 1994 Attractors for random dynamical systems Probab. Theory Related Fields 100 365-93

[12] Franks J 1988 A variation of Poincaré-Birkhoff theorem Hamiltonian Dynamical Systems (Contemporary Mathematics vol 81) (Providence: Amer. Math. Soc.) pp. 111-17

[13] Hurley M 1991 Chain recurrence and attraction in non-compact spaces Ergod. Th. Dynam. Sys. 11 709-29

[14] Hurley M 1992 Noncompact chain recurrence and attraction Proc. Amer. Math. Soc. 115 1139-48

[15] Hurley M 1995 Chain recurrence, semiflows, and gradients J. Dyn. Diff. Equ. 7 437-56

[16] Kloeden P E and Platen E 1992 Numerical Solution of Stochastic Differential Equations (Berlin Heidelberg New York: Springer-Verlag)

[17] Ochs G 1999 Weak Random Attractors Institut für Dynamische Systeme, Universität Bremen Report 449

[18] Scheutzow M 2002 Comparison of various concepts of a random attractor: A case study Arch. Math. 78 233-40

[19] Schmalfuss B 1992 Backward cocycles and attractors for stochastic differential equations In: Reitmann V, Riedrich T and Koksch N (Eds.) International Seminar on Applied Mathematics - Nonlinear Dynamics: Attractor Approximation and Global Behaviour (Teubner, Leipzig) pp. 185-92

[20] Schmalfuss B 1997 The random attractor of the stochastic Lorenz system ZAMP 48 951-75 